\title{
Hermiticity and the Cohomology Condition in Topological Yang-Mills Theory*
}

\author{
Jean-Guy Demers ${ }^{\dagger}$ \\ Center for Theoretical Physics, \\ Laboratory for Nuclear Science \\ and Department of Physics \\ Massachusetts Institute of Technology, Cambridge, MA 02139
}

\begin{abstract}
The symmetries of the topological Yang-Mills theory are studied in the Hamiltonian formalism and the generators of the twisted $\mathrm{N}=2$ superPoincaré algebra are explicitly constructed. Noting that the twisted Lorentz generators do not generate the Lorentz symmetry of the theory, we relate the two by extracting from the latter the twisted version of the internal SU(2) generator. The hermiticity properties of the various generators are also considered throughout, and the boost generators are found to be non-hermitian. We then recover the BRST cohomology condition on physical states from representation theory arguments.
\end{abstract}

CTP\#2233

September 1993

\footnotetext{
†jgdemers@mitlns.mit.edu

*This work is supported in part by funds provided by the U. S. Department of Energy (D.O.E.) under contract \#DE-AC02-76ER03069.
} 


\section{INTRODUCTION}

In recent years, much attention has been devoted to the study of topological field theories [1]. Because these theories have no local dynamics, their correlation functions depend only on the global features of the target space. An important example is given by the topological Yang-Mills (TYM) theory, which was used to obtain the Donaldson invariants for smooth 4-manifolds [2]. Shortly after TYM was introduced, it was shown [3, [1] that it can also be obtained by BRST gauge fixing the topological symmetry $\left(\delta A_{\alpha}^{a}=\theta_{\alpha}^{a}\right.$, with $\theta_{\alpha}^{a}$ arbitrary) of either zero or the topological action $S=\int d^{4} x F^{\mu \nu} \tilde{F}_{\mu \nu}$. Under an appropriate choice of gauge parameters, the resulting action is identical to the one introduced in [2] and is given by:

$$
\begin{aligned}
S= & \int_{M} \operatorname{Tr}\left\{\frac{1}{4} F_{\alpha \beta} F^{\alpha \beta}-\frac{1}{2} D_{\alpha} \phi D^{\alpha} \lambda-i \eta D_{\alpha} \psi^{\alpha}+i D_{\alpha} \psi_{\beta} \chi^{\alpha \beta}\right. \\
& \left.\quad-\frac{i}{8} \phi\left[\chi_{\alpha \beta}, \chi^{\alpha \beta}\right]-\frac{i}{2} \lambda\left[\psi_{\alpha}, \psi^{\alpha}\right]-\frac{i}{2} \phi[\eta, \eta]-\frac{1}{8}([\phi, \lambda])^{2}\right\} \\
\equiv \int_{M} \mathcal{L} &
\end{aligned}
$$

Here, all fields are Lie algebra valued and transform according to the adjoint representation of the gauge group, which is taken to be compact and semi-simple. The covariant derivative is $D_{\alpha}=\nabla_{\alpha}+\left[A_{\alpha},\right]$, where $\nabla_{\alpha}$ is the covariant derivative with respect to the diffeomorphisms on the curved manifold $M$ of metric $g_{\mu \nu}$. The gauge field $A_{\alpha}$ and the scalars $\lambda$ and $\phi$ are bosonic while $\eta, \psi_{\alpha}$ and $\chi_{\alpha \beta}$ are all anticommuting and respectively scalar, vector and selfdual tensor fields $\left(\chi_{\alpha \beta}=\frac{1}{2} \varepsilon_{\alpha \beta}{ }^{\mu \nu} \chi_{\mu \nu}\right)$. Note that in this version (11) still possesses the usual (non-topological) Yang-Mills symmetry.

When (1) was introduced, its intimate relation with $N=2$ super Yang-Mills (SYM) was already noticed [2]. In fact, formal representations of the Donaldson polynomials have also been obtained in SYM [11]. In Euclidean space-time, the latter theory enjoys the Lorentz symmetry $S O(4)$ (isomorphic to $S U_{L}(2) \otimes S U_{R}(2)$ ) as well as the internal global $S U_{I}(2)$ symmetry. If one "twists" this symmetry by replacing $S U_{L}(2)$ by the diagonal sum of $S U_{L}(2)$ and $S U_{I}(2), S U_{L^{\prime}}(2)$, the rotation group then becomes $S U_{L^{\prime}}(2) \otimes S U_{R}(2)$ and the 
resulting theory is just (1). Through this procedure the original supersymmetry generators are also transformed and the Lorentz scalar supercharge thus obtained is identified as the BRST charge. The twist procedure has also been used to obtain extended $(N=2)$ TYM theories [5]. Furthermore, TYM has also been obtained via the use of Killing spinors in $N=2$ conformal supergravity [6]; in this case, a "local" version of the twisting procedure is implemented by embedding the $S U(2)$ connection in the Lorentz spin connection.

In this paper, we will detail the twisting of the $N=2$ supersymmetry (Section 【I) and explicitly construct the various generators while studying their hermiticity properties (Section [II). We will argue that after twisting, the internal symmetry generators are transformed into a useful and hitherto unappreciated symmetry of (1). It will also be shown that

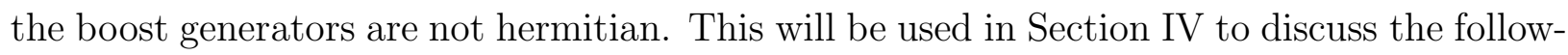
ing issue. Despite their connection through twisting, TYM and SYM theories differ in that the former does not support any local excitations. When TYM is considered through the BRST construction, it is found that the only states in the cohomology of the BRST charge are those with vanishing energy [2]. Among other things, this absence of local excitations complicates any attempt to use topological field theories in a description of quantum gravity, a possibility suggested by the natural general covariance of these theories. It is hence usually thought that one must first establish a mechanism to break the topological symmetry. As a consequence, we find it compelling to study more closely the relation between SYM and TYM. Within the context of twisting and without appealing to the BRST derivation of (1), we will propose an explanation, based on representation theory arguments, of why TYM is indeed free of local excitations. As we are only interested in the details of the canonical quantization of the theory, such as its hermiticity properties and spectrum, we will work on flat manifolds. Our concluding remarks are contained in Section $\nabla$.

\section{TWISTED N=2 SUPERSYMMETRY ALGEBRA}

Our starting point is the $N=2$ superPoincaré algebra (without central charge) [7, [8]: 


$$
\begin{aligned}
{\left[P_{\alpha}, P_{\beta}\right] } & =0, \quad\left[P_{\mu}, J_{\alpha \beta}\right]=i g_{\mu[\alpha} P_{\beta]}, \\
{\left[J_{\alpha \beta}, J_{\mu \nu}\right] } & =i g_{\alpha[\mu} J_{\nu] \beta}+i g_{\beta[\mu} J_{\alpha \mid \nu]}, \\
{\left[P_{\mu}, Q_{A i}\right] } & =0=\left[P_{\mu}, \bar{Q}_{\dot{A} j}\right], \\
{\left[Q_{A i}, J_{\alpha \beta}\right] } & =\left(\sigma_{\alpha \beta}\right)_{A}{ }^{B} Q_{B i}, \quad\left[\bar{Q}^{\dot{A}}{ }_{j}, J_{\alpha \beta}\right]=\left(\bar{\sigma}_{\alpha \beta}\right)^{\dot{A}}{ }_{\dot{B}} \bar{Q}^{\dot{B}}{ }_{j}, \\
\left\{Q_{A i}, \bar{Q}_{\dot{B}}{ }^{j}\right\} & =2 \delta_{i}{ }^{j} P_{\alpha}\left(\sigma^{\alpha}\right)_{A \dot{B}}, \\
\left\{Q_{A i}, Q_{B j}\right\} & =0=\left\{\bar{Q}_{\dot{A}}{ }^{i}, \bar{Q}_{\dot{B}}{ }^{j}\right\}, \\
{\left[T^{i}{ }_{j}, J_{\alpha \beta}\right] } & =0=\left[T^{i}{ }_{j}, P_{\alpha}\right], \\
{\left[T^{i}{ }_{j}, T^{k}{ }_{l}\right] } & =\frac{1}{2}\left(\delta_{j}^{k} T^{i}{ }_{l}-\delta^{i}{ }_{l} T^{k}{ }_{j}\right), \\
{\left[T^{i}{ }_{j}, Q_{A k}\right] } & =-\frac{1}{2}\left(\delta_{k}^{i} Q_{A j}-\frac{1}{2} \delta_{j}^{i} Q_{A k}\right), \\
{\left[T^{i}{ }_{j}, \bar{Q}_{\dot{A} k}\right] } & =-\frac{1}{2}\left(\delta_{k}^{i} \bar{Q}_{\dot{A} j}-\frac{1}{2} \delta_{j}^{i} \bar{Q}_{\dot{A} k}\right) .
\end{aligned}
$$

Our convention closely follows Ref. [9]. Bracketed indices are to be antisymmetrized, ignoring the ones just preceeding a vertical bar (thus $g_{\beta[\mu} J_{\alpha \mid \nu]} \equiv g_{\beta \mu} J_{\alpha \nu}-g_{\beta \nu} J_{\alpha \mu}$ ). Greek letters denote Lorentz indices, with $P_{\alpha}$ and $J_{\alpha \beta}$ standing for translation and Lorentz generators respectively. Capital latin letters are two-spinor indices with undotted ones referring to $S U_{R}(2)$ and dotted ones to $S U_{L}(2)$. Raising and lowering these indices is done with the help of the antisymmetric matrices $\varepsilon_{A B}, \varepsilon^{A B}, \varepsilon_{\dot{A} \dot{B}}$ and $\varepsilon^{\dot{A} \dot{B}}$. They are given by: $\varepsilon_{12}=-\varepsilon^{12}=\varepsilon_{\dot{1} \dot{2}}=-\varepsilon^{i \dot{2}}=-1$ and act on Weyl spinors as: $\bar{\psi}_{\dot{A}}=\varepsilon_{\dot{A} \dot{B}} \bar{\psi}^{\dot{B}}, \chi^{A}=\varepsilon^{A B} \chi_{B}$. The internal indices are $i, j \ldots$ (in subsequent sections, these symbols will be used as spatial components of Lorentz indices); $T_{j}^{i}$ is traceless and generate $S U_{I}(2)$. The metric $g_{\alpha \beta}$ is euclidean $\left(=\delta_{\alpha \beta}\right)$ whereas $\sigma_{\alpha}=\left(-i, \sigma_{j}\right), \bar{\sigma}_{\alpha}=\left(i, \sigma_{j}\right)$ where $\sigma_{i}$ are the usual Pauli matrices. Similarly to the Minkowski case, we define $\sigma_{\alpha \beta}=\frac{i}{4}\left(\sigma_{\alpha} \bar{\sigma}_{\beta}-\sigma_{\beta} \bar{\sigma}_{\alpha}\right)$ and $\bar{\sigma}_{\alpha \beta}=\frac{i}{4}\left(\bar{\sigma}_{\alpha} \sigma_{\beta}-\bar{\sigma}_{\beta} \sigma_{\alpha}\right)$.

We now perform the twisting of this algebra. Replacing $S U_{L}(2)$ by the diagonal sum of $S U_{L}(2)$ and $S U_{I}(2)$ translates into the identification of the internal indices with left handed Weyl spinor indices, leading to:

$$
\begin{aligned}
& Q_{A i} \rightarrow Q_{A \dot{C}}=a_{1}\left(\sigma^{\alpha}\right)_{A \dot{C}} \bar{Q}_{\alpha} \\
& \bar{Q}_{j}^{\dot{B}} \rightarrow \bar{Q}_{\dot{D}}^{\dot{B}}=a_{2} \delta_{\dot{D}}^{\dot{B}} Q_{+} a_{3}\left(\bar{\sigma}^{\mu \nu}\right)_{\dot{D}}^{\dot{B}} S_{\mu \nu}
\end{aligned}
$$




$$
T^{i}{ }_{j} \rightarrow T_{\dot{B}}^{\dot{A}}=a_{4}\left(\bar{\sigma}^{\mu \nu}\right)^{\dot{A}}{ }_{\dot{B}} R_{\mu \nu},
$$

where on the RHS, the twisted quantities are expressed in terms of their Lorentz components: $\bar{Q}_{\alpha}$ is a vector, $Q$ a scalar, whereas $S_{\mu \nu}$ and $R_{\mu \nu}$ are self-dual tensors; $a_{i}$ 's are arbitrary constants. At this stage, these constants could be absorbed in the definition of the generators, but they will be useful in the next section, as we will use already known expressions for $Q$ and $\bar{Q}_{\alpha} . Q, \bar{Q}_{\alpha}$ and $S_{\mu \nu}$ are Grassman odd, whereas $R_{\mu \nu}$ is Grassman even. Note that vectorial Grassman charges are also known to exist in non-critical string theory [10]. The relations (3) can be inverted:

$$
\begin{aligned}
\bar{Q}_{\alpha} & =\frac{1}{2 a_{1}}\left(\sigma_{\alpha}\right)^{\dot{C} A} Q_{A \dot{C}}, \\
Q & =\frac{1}{2 a_{2}} \delta_{\dot{B}}^{\dot{D}} \bar{Q}^{\dot{B}} \dot{D}, \\
S_{\mu \nu} & =\frac{1}{2 a_{3}}\left(\bar{\sigma}_{\mu \nu}\right)^{\dot{B}}{ }_{\dot{D}} \bar{Q}^{\dot{D}}{ }_{\dot{B}}, \\
R_{\mu \nu} & =\frac{1}{2 a_{4}}\left(\bar{\sigma}_{\mu \nu}\right)^{\dot{A}}{ }_{\dot{B}} T^{\dot{B}}{ }_{\dot{A}},
\end{aligned}
$$

where we have made use of the identity:

$$
\operatorname{Tr}\left(\bar{\sigma}_{\alpha \beta} \bar{\sigma}_{\mu \nu}\right)=\frac{1}{2} \delta_{\alpha[\mu} \delta_{\nu] \beta}+\frac{1}{2} \epsilon_{\alpha \beta \mu \nu} .
$$

Under the twisting (3), the superPoincaré algebra (2) is transformed into:

$$
\begin{aligned}
{\left[P_{\alpha}, P_{\beta}\right] } & =0, \quad\left[P_{\mu}, J_{\alpha \beta}\right]=i \delta_{\mu[\alpha} P_{\beta]}, \\
{\left[J_{\alpha \beta}, J_{\mu \nu}\right] } & =i \delta_{\alpha[\mu} J_{\nu] \beta}+i \delta_{\beta[\mu} J_{\mid \alpha \nu]}, \\
{\left[P_{\beta}, Q\right] } & =\left[P_{\beta}, \bar{Q}_{\alpha}\right]=\left[P_{\beta}, S_{\mu \nu}\right]=0, \\
{\left[Q, J_{\alpha \beta}\right] } & =\frac{a_{3}}{a_{2}} S_{\alpha \beta}, \\
{\left[\bar{Q}_{\mu}, J_{\alpha \beta}\right] } & =\frac{i}{2}\left(\delta_{\mu[\alpha} S_{\beta] \nu}-\delta_{\nu[\alpha} S_{\beta] \mu}\right), \\
{\left[S_{\mu \nu}, J_{\alpha \beta}\right] } & =\frac{Q}{4} \frac{a_{2}}{a_{3}}\left(\delta_{\alpha[\mu} \delta_{\nu] \beta}+\epsilon_{\alpha \beta \mu \nu}\right)+\frac{i}{2}\left(\delta_{\mu[\alpha} S_{\beta] \nu}-\delta_{\nu[\alpha} S_{\beta] \mu}\right), \\
\left\{Q, \bar{Q}_{\alpha}\right\} & =-\frac{1}{a_{1} a_{2}} P_{\alpha}, \\
\left\{\bar{Q}_{\alpha}, S_{\mu \nu}\right\} & =-\frac{i}{2 a_{1} a_{3}}\left(\delta_{\alpha[\mu} P_{\nu]}+\epsilon_{\alpha \mu \nu \beta} P^{\beta}\right),
\end{aligned}
$$




$$
\begin{aligned}
\left\{\bar{Q}_{\alpha}, \bar{Q}_{\beta}\right\} & =\{Q, Q\}=\left\{S_{\mu \nu}, S_{\alpha \beta}\right\}=0 \\
{\left[Q, R_{\mu \nu}\right] } & =\frac{a_{3}}{4 a_{2} a_{4}} S_{\mu \nu}, \\
{\left[R_{\mu \nu}, S_{\alpha \beta}\right] } & =-\frac{a_{2}}{16 a_{3} a_{4}} Q\left(\delta_{\alpha[\mu} \delta_{\nu] \beta}+\epsilon_{\alpha \beta \mu \nu}\right)-\frac{i}{8 a_{4}}\left(\delta_{\mu[\alpha} S_{\beta] \nu}-\delta_{\nu[\alpha} S_{\beta] \mu}\right), \\
{\left[\bar{Q}_{\alpha}, R_{\mu \nu}\right] } & =-\frac{i}{8 a_{4}}\left(\delta_{\alpha[\mu} \bar{Q}_{\nu]}+\varepsilon_{\mu \nu \alpha \beta} \bar{Q}^{\beta}\right), \\
{\left[R_{\mu \nu}, R_{\alpha \beta}\right] } & =-\frac{i}{4 a_{4}}\left(\delta_{\mu[\alpha} R_{\beta] \nu}-\delta_{\nu[\alpha} R_{\beta] \mu}\right), \\
{\left[R_{\mu \nu}, J_{\alpha \beta}\right] } & =0=\left[R_{\mu \nu}, P_{\beta}\right] .
\end{aligned}
$$

The existence of (6) was conjectured in 11, 12. The Poincaré sector of the algebra Eqs. (6a

- 6b) is of course left unchanged by the twisting. This would suggest that for the twisted theory, $J_{\alpha \beta}$ also generate Lorentz rotations. However, a look at (6) reveals that the fermionic charges, as well as $R_{\mu \nu}$, do not transform in the expected way (e.g. $Q$ does not transform as a scalar). In the following section, we will examine how the algebra (6) is realized in TYM, and identify the correct Lorentz generators.

\section{THE ALGEBRA REALIZED}

In order to study the twisted $N=2$ superPoincaré symmetries of (1), we make use of Noether's theorem in its Lagrangian form. Under a symmetry transformation, the variation of the Lagrangian density is a total derivative $\delta \mathcal{L}=\partial_{\mu} \Lambda^{\mu}$ and using the equations of motion, the current $J^{\mu}=\sum_{\text {fields } \Phi} \delta \Phi \frac{\partial \mathcal{L}}{\partial\left(\partial_{\mu} \Phi\right)}-\Lambda^{\mu}$ is conserved 13. The simplest of the symmetries is the invariance under translation, for which $\delta \mathcal{L}=a^{\mu} \partial_{\mu} \mathcal{L}$ with $a^{\mu}$ a constant infinitesimal parameter. The corresponding form of the energy-momentum tensor is given by:

$$
\begin{gathered}
\theta^{\alpha \gamma}=F_{\mu}^{\gamma} F^{\alpha \mu}-\frac{1}{2} D^{\alpha} \phi D^{\gamma} \lambda-\frac{1}{2} D^{\alpha} \lambda D^{\gamma} \phi+i D^{\gamma} \psi^{\alpha} \eta+i D^{\gamma} \psi_{\mu} \chi^{\alpha \mu} \\
+D_{\mu} A^{\gamma} F^{\alpha \mu}+A^{\gamma} J^{\alpha}-g^{\alpha \gamma} \mathcal{L}
\end{gathered}
$$

with

$$
J_{\alpha}=\frac{1}{2}\left[\phi, D_{\alpha} \lambda\right]+\frac{1}{2}\left[\lambda, D_{\alpha} \phi\right]-i\left[\psi_{\alpha}, \eta\right]-i\left[\psi^{\mu}, \chi_{\alpha \mu}\right]
$$


and where $\mathcal{L}$ is the Lagrangian density given in (11). The conservation of this tensor, $\partial_{\alpha} \theta^{\alpha \gamma}=$ 0 , gives rise to the energy and momentum generators:

$$
\begin{gathered}
P_{0}=\int d^{3} x\left\{\frac{1}{2}\left(F_{0 i} F_{0 i}-\tilde{F}_{0 i} \tilde{F}_{0 i}\right)-\frac{1}{2} D_{0} \phi D_{0} \lambda+\frac{1}{2} D_{i} \phi D_{i} \lambda-i \varepsilon_{i j k} D_{j} \psi_{k} \chi_{i}\right. \\
-i \psi_{0} D_{i} \chi_{i}+i \eta D_{i} \psi_{i}+\frac{i}{2} \phi\left[\chi_{i}, \chi_{i}\right]+\frac{i}{2} \lambda\left[\psi_{i}, \psi_{i}\right] \\
\left.+\frac{i}{2} \lambda\left[\psi_{0}, \psi_{0}\right]+\frac{i}{2} \phi[\eta, \eta]+\frac{1}{8}([\phi, \lambda])^{2}-A_{0} G\right\}, \\
P_{i}=\int d^{3} x\left\{F_{0 k} F_{i k}-\frac{1}{2} D_{0} \lambda D_{i} \phi-\frac{1}{2} D_{0} \phi D_{i} \lambda+i D_{i} \psi_{j} \chi_{j}+i D_{i} \eta \psi_{0}-A_{i} G\right\},
\end{gathered}
$$

where $\chi_{i} \equiv \chi_{0 i}$. Here and in the rest, integrations are over "spatial" coordinates, with traces understood. We also ignore ordering ambiguities. In Eq. (9), $A_{0}$ should be viewed as the Lagrange multiplier which imposes the generalized Gauss law constraint $G \equiv D_{i} F_{0 i}-J_{0} \approx 0$. The Hamiltonian (9) can equally be obtained by Legendre transforming (11). In order to compute the algebra of these charges, we first identify the various momenta of (11), and impose on them the appropriate equal-time canonical commutators:

$$
\begin{array}{rlrl}
P_{\psi_{i}^{a}} & =i \chi_{i}^{a}, \\
P_{\psi_{0}^{a}} & =i \eta^{a}, & \left\{\chi_{i}^{a}(x), \psi_{j}^{b}(y)\right\} & =-\delta^{a b} \delta_{i j} \delta(x-y), \\
P_{A_{i}^{a}} & =F_{0 i}^{a}, \\
P_{\phi^{a}} & =-\frac{1}{2}\left(D_{0}^{a}(x), \eta^{b}(y)\right\}=-\delta^{a b} \delta(x-y), \\
P_{\lambda^{a}} & =-\frac{1}{2}\left(D_{0} \phi\right)^{a}, & {\left[A_{i}^{a}(x), F_{0 j}^{b}(y)\right]} & =i \delta^{a b} \delta_{i j} \delta(x-y), \\
{\left[\phi^{a}(x),-\frac{1}{2}\left(D_{0} \lambda\right)^{b}(y)\right]} & =i \delta^{a b} \delta(x-y), \\
& {\left[\lambda^{a}(x),-\frac{1}{2}\left(D_{0} \phi\right)^{b}(y)\right]} & =i \delta^{a b} \delta(x-y),
\end{array}
$$

where $\mathrm{x}$ and $\mathrm{y}$ denote here space coordinates. Making use of these commutators, $P_{0}$ and $P_{i}$ are found to correctly translate the fields, and when commuted among themselves yield:

$$
\left[P_{i}, P_{j}\right]=0, \quad\left[P_{0}, P_{j}\right]=i \int d^{3} x \partial_{j} A_{0}(x) G(x)
$$

As with various forthcoming commutators, we find that because of the remnant Yang-Mills symmetry in the action (四), the algebra (6) is only realized on physical states, annihilated by the constraint $G$. 
We now wish to study the hermiticity properties of our generators. We take for adjoint assignments:

$$
\begin{aligned}
& A_{\alpha}^{\dagger}=A_{\alpha}, \\
& \psi_{i}^{\dagger}=-\chi_{i}, \\
& \psi_{0}^{\dagger}=\eta, \\
& \phi^{\dagger}=\lambda .
\end{aligned}
$$

Despite its non-covariance, this choice is natural for various reasons. In order for the field theory to be well defined, $P_{0}$ should be hermitian and it is under (13). Moreover, as is shown below, this choice also leads to a semi-positive definite spectrum for $P_{0}$, in analogy with SYM theory. Also, $P_{i}$ and the Lagrangian (1) are equally hermitian with this prescription. Note that because of the peculiarity of the self-duality operation in euclidean metric [14] ( $\chi_{\alpha \beta}=\frac{1}{2 \lambda} \varepsilon_{\alpha \beta \mu \nu} \chi^{\mu \nu}$ with $\lambda=1, i$ for euclidean and Minkowskian metrics respectively), we require $\varepsilon_{\alpha \beta \mu \nu}$ to change sign when taking the adjoint. Given that the presence of $\varepsilon_{\alpha \beta \mu \nu}$ in the various generators has its origin in the self-dualtiy of $\chi_{\alpha \beta}$, this prescription in effect reproduce the study of hermiticity in Minkowskian metric. An alternative road would be to study the Lagrangian (11) in Minkowski spacetime. The symmetry generators would then, up to signs, be the same as the ones presented here for euclidean metric. The algebra of the generators in that case would be a Wick rotated version of (6), obtained by the change: $\delta_{\mu \nu} \rightarrow \eta_{\mu \nu}(\equiv \operatorname{diag}(-1,1,1,1)), \sigma^{\alpha} \rightarrow\left(1, \sigma_{i}\right), \bar{\sigma}^{\alpha} \rightarrow\left(-1, \sigma_{i}\right), \varepsilon_{\alpha \beta \mu \nu} \rightarrow \frac{1}{i} \varepsilon_{\alpha \beta \mu \nu}$.

Under Lorentz transformations, the variation of the fields is $\delta \Phi=\omega^{\mu \nu} x_{\mu} \partial_{\nu} \Phi$ where $\omega^{\mu \nu}$ is an infinitesimal antisymmetric parameter. The corresponding currents:

$$
\begin{aligned}
M^{\alpha \beta \gamma}= & {\left[x ^ { \beta } \left\{-\frac{1}{2} D^{\alpha} \lambda D^{\gamma} \phi-\frac{1}{2} D^{\alpha} \phi D^{\gamma} \lambda+F_{\mu}^{\gamma} F^{\alpha \mu}+i D^{\gamma} \eta \psi^{\alpha}+i D^{\gamma} \psi_{\mu} \chi^{\alpha \mu}\right.\right.} \\
& \left.+\frac{A^{\gamma}}{2}\left[\lambda, D^{\alpha} \phi\right]+\frac{A^{\gamma}}{2}\left[\phi, D^{\alpha} \lambda\right]-i A^{\gamma}\left[\eta, \psi^{\alpha}\right]-i A^{\gamma}\left[\psi_{\mu}, \chi^{\alpha \mu}\right]-g^{\alpha \gamma} \mathcal{L}\right\} \\
& \left.+D_{\mu}\left(x^{\beta} A^{\gamma}\right) F^{\alpha \mu}+i \psi^{\gamma} \chi^{\alpha \beta}\right]-[\beta \leftrightarrow \gamma]
\end{aligned}
$$

are conserved $\left(\partial_{\alpha} M^{\alpha \beta \gamma}=0\right)$ and lead to the constants of motion associated with boosts and rotations: 


$$
\begin{gathered}
M_{0 i}=x_{0} P_{i}-\int d^{3} x x_{i} \mathcal{P}_{0}+i \int d^{3} x \psi_{i} \eta, \\
M_{k j}=\int d^{3} x\left\{\left(x_{k} \mathcal{P}_{j}-i \psi_{k} \chi_{j}\right)-(k \leftrightarrow j)\right\},
\end{gathered}
$$

where $\mathcal{P}_{0}$ is the energy density, as integrated in Eq.(9) and similarly for $\mathcal{P}_{j}$ from Eq.(10). One can readily check, using (13), that $M_{k j}$ is hermitian but that $M_{0 i}$ is not. We will return to this point in Section $\mathbb{I V}$, in connection with the possible excited states of the theory. Using Eq.(11), the following commutators are obtained:

$$
\begin{gathered}
{\left[P_{0}, M_{0 i}\right]=i x_{0} \int d^{3} x \partial_{i} A_{0}(x) G(x)+i \int d^{3} x A_{i}(x) G(x)+i P_{i}, \quad\left[P_{0}, M_{k j}\right]=0,} \\
{\left[P_{j}, M_{0 i}\right]=-i \delta_{i j} P_{0}, \quad\left[P_{i}, M_{k j}\right]=i \delta_{i[k} P_{j]},} \\
{\left[M_{0 i}, M_{0 j}\right]=-i M_{i j},} \\
{\left[M_{k j}, M_{l m}\right]=i \delta_{k[l} M_{m] j}+i \delta_{j[l} M_{k \mid m]} .}
\end{gathered}
$$

Together with (12), we thus recover the Poincaré sector of (6).

Turning to the twisted supersymmetries, we have the scalar charge Q, identified in [2]. It is preserved on an arbitrary manifold and the energy momentum tensor can by expressed as a $Q$ variation. In the context of the BRST construction of (1), it is precisely the BRST charge. Its expression is:

$$
Q=\int d^{3} x\left\{\left(F_{0 i}+\tilde{F}_{0 i}\right) \psi_{i}-\eta D_{0} \phi-D_{i} \phi \chi_{i}-\frac{\psi_{0}}{2}[\lambda, \phi]\right\}
$$

Under translation and rotation, it transforms as:

$$
\begin{gathered}
{\left[P_{0}, Q\right]=0=\left[P_{j}, Q\right],} \\
{\left[M_{0 i}, Q\right]=i \int d^{3} x x_{i} \psi_{0}(x) G(x), \quad\left[M_{k j}, Q\right]=0,} \\
\frac{1}{2}\{Q, Q\}=-\int d^{3} x \phi(x) G(x) .
\end{gathered}
$$


We thus recover the nilpotency of $Q$ (up to gauge transformations), but (23) shows that $M_{\alpha \beta}$ does not correspond to the generator $J_{\alpha \beta}$ appearing in (6). This is confirmed by the study of $\bar{Q}_{\alpha}$, also identified in [2]. Its time and space components are given by:

$$
\begin{gathered}
\bar{Q} \equiv \bar{Q}_{0}=\int d^{3} x\left\{\left(F_{0 i}-\tilde{F}_{0 i}\right) \chi_{i}+\psi_{0} D_{0} \lambda-\psi_{i} D_{i} \lambda+\frac{\eta}{2}[\phi, \lambda]\right\} \\
\bar{Q}_{i}=\int d^{3} x\left\{\varepsilon_{i j k}\left(F_{0 j}-\tilde{F}_{0 j}\right) \chi_{k}+\psi_{0} D_{i} \lambda+\psi_{i} D_{0} \lambda-\left(F_{0 i}-\tilde{F}_{0 i}\right) \eta+\varepsilon_{i j k} \psi_{j} D_{k} \lambda+\frac{1}{2}[\phi, \lambda] \chi_{i}\right\} .
\end{gathered}
$$

The spacetime symmetry transformations of $\bar{Q}$ and $\bar{Q}_{i}$ are made clear by:

$$
\begin{array}{cc}
{\left[P_{0}, \bar{Q}\right]=-i \int d^{3} x \eta(x) G(x),} & {\left[P_{i}, \bar{Q}\right]=0,} \\
{\left[M_{0 i}, \bar{Q}\right]=i \bar{Q}_{i}-i \int d^{3} x x_{i} \eta(x) G(x),} & {\left[M_{k j}, \bar{Q}\right]=0,} \\
\frac{1}{2}\{\bar{Q}, \bar{Q}\}=-\int d^{3} x \lambda(x) G(x), &
\end{array}
$$

as well as:

$$
\begin{array}{cc}
{\left[P_{0}, \bar{Q}_{i}\right]=-i \int d^{3} x \chi_{i}(x) G(x),} & {\left[P_{j}, \bar{Q}_{i}\right]=0,} \\
{\left[M_{0 i}, \bar{Q}_{j}\right]=i \delta_{i j} \bar{Q},} & {\left[M_{k j}, \bar{Q}_{i}\right]=i \delta_{i[j} \bar{Q}_{k]},} \\
\frac{1}{2}\left\{\bar{Q}_{i}, \bar{Q}_{j}\right\}=-\delta_{i j} \int d^{3} x \lambda(x) G(x), & \frac{1}{2}\left\{\bar{Q}, \bar{Q}_{i}\right\}=0
\end{array}
$$

When $\bar{Q}_{\alpha}$ is anticommuted with the BRST generator, it gives:

$$
\frac{1}{2}\left\{Q, \bar{Q}_{\alpha}\right\}=-P_{\alpha}-\int d^{3} x A_{\alpha}(x) G(x)
$$

Thus, given our choice of generators, (21) (25) and (26), the relation (6g) is obtained, provided $a_{1} a_{2}=\frac{1}{2}$. Observe how the adjoint assignments (13) produce:

$$
Q^{\dagger}=-\bar{Q}
$$


and as announced they render the Hamiltonian (9) semi-positive definite (as is the case in SYM).

To identify $S_{\mu \nu}$, we compute the adjoint of $\bar{Q}_{i}$, obtaining:

$S_{0 i}=\int d^{3} x\left[\varepsilon_{i j k}\left(F_{0 j}+\tilde{F}_{0 j}\right) \psi_{k}+\eta D_{i} \phi-\chi_{i} D_{0} \phi-\left(F_{0 i}+\tilde{F}_{0 i}\right) \psi_{0}+\varepsilon_{i j k} \chi_{j} D_{k} \phi-\frac{1}{2}[\lambda, \phi] \psi_{i}\right]$.

Its spacetime symmetry transformations and nilpotency are revealed by the following set of commutators:

$$
\begin{array}{cc}
{\left[P_{0}, S_{0 i}\right]=-i \int d^{3} x \psi_{i}(x) G(x),} & {\left[P_{j}, S_{0 i}\right]=0} \\
{\left[M_{0 i}, S_{0 j}\right]=-i \varepsilon_{i j k} S_{0 k},} & {\left[M_{k j}, S_{0 i}\right]=i \delta_{i[j} S_{0 \mid k]},} \\
\frac{1}{2}\left\{S_{0 i}, S_{0 j}\right\}=-\delta_{i j} \int d^{3} x \phi(x) G(x) .
\end{array}
$$

Eqs.(36) and (37) show that $S_{0 i}$ generates a symmetry if Gauss's law is imposed and that it is a self-dual object. Relating to previous fermionic symmetries, we compute:

$$
\begin{gathered}
\left\{S_{0 i}, Q\right\}=0, \\
\frac{1}{2}\left\{S_{0 i}, \bar{Q}\right\}=P_{i}+\int d^{3} x A_{i}(x) G(x), \\
\frac{1}{2}\left\{S_{0 i}, \bar{Q}_{j}\right\}=-\delta_{i j}\left(P_{0}+\int d^{3} x A_{0}(x) G(x)\right)+\varepsilon_{i j k}\left(P_{k}+\int d^{3} x A_{k}(x) G(x)\right),
\end{gathered}
$$

which reproduces (6h), if $a_{1} a_{3}=\frac{-i}{4}$.

Noting now that the boost generators are not hermitian, we extract from them the twisted internal generators by taking the anti-hermitian part $R_{0 i} \equiv M_{0 i}^{\dagger}-M_{0 i}$. In terms of the fields, it is simply:

$$
R_{0 i}=\int d^{3} x\left(-i \psi_{i} \eta+i \psi_{0} \chi_{i}+i \varepsilon_{i l m} \psi_{l} \chi_{m}\right)
$$


Commuting with the Poincaré generators produces:

$$
\begin{array}{cc}
{\left[P_{0}, R_{0 i}\right]=0,} & {\left[P_{j}, R_{0 i}\right]=0,} \\
{\left[M_{0 i}, R_{0 j}\right]=-i \varepsilon_{i j k} R_{0 k},} & {\left[M_{k j}, R_{0 i}\right]=i \delta_{i[j} R_{0 \mid k]},}
\end{array}
$$

which shows that $R_{0 i}$ is also a self-dual object. When commuted with the fermionic symmetries and with itself, we get:

$$
\begin{gathered}
{\left[R_{0 i}, Q\right]=i S_{0 i}, \quad\left[R_{0 i}, \bar{Q}\right]=i \bar{Q}_{i}} \\
{\left[R_{0 i}, \bar{Q}_{j}\right]=i \varepsilon_{i j k} \bar{Q}_{k}-i \delta_{i j} \bar{Q}, \quad\left[R_{0 i}, S_{0 j}\right]=i \varepsilon_{i j k} S_{0 k}-i \delta_{i j} Q} \\
{\left[R_{0 i}, R_{0 j}\right]=2 i \varepsilon_{i j k} R_{0 k}}
\end{gathered}
$$

We thus find that (6) is realized in TYM with the following values of parameters: $a_{1}=$ $1, a_{2}=\frac{1}{2}, a_{3}=\frac{-i}{4}$ and $a_{4}=\frac{1}{8}$. As an infinitesimal transformation, $R_{\alpha \beta}$ only acts on fermionic fields (as is obvious from (42) and in parallel with SYM) :

$$
\begin{aligned}
\delta_{R} \eta & =\frac{1}{2} \zeta_{\rho \sigma} \chi^{\rho \sigma}, \\
\delta_{R} \psi_{\alpha} & =-2 \zeta_{\alpha \lambda} \psi^{\lambda}, \\
\delta_{R} \chi_{\alpha \beta} & =2 \zeta_{\alpha \beta} \eta-\zeta_{\lambda[\alpha} \chi_{\beta]}{ }^{\lambda},
\end{aligned}
$$

where $\zeta_{\alpha \beta}$ is an infinitesimal, commuting and self-dual parameter. Although relatively simple, this symmetry appears to have escaped notice. It would be interesting to investigate its use, for instance, in the perturbative renormalization of TYM [3, 15] or determine the class of manifolds on which it is preserved [12].

So far, we have thus identified for TYM all the generators in the twisted $N=2$ superalgebra (6), with the exception of $J_{\alpha \beta}$. This generator should be hermitian, since it is so before twisting. The more or less natural object to consider here is the hermitian part of $M_{0 i}$. So we conjecture: 


$$
\begin{aligned}
& J_{0 i}=M_{0 i}+\frac{R_{0 i}}{2}, \\
& J_{k j}=M_{k j}+\frac{1}{2} \varepsilon_{k j l} R_{0 l},
\end{aligned}
$$

where in (49b), we have used the self-duality of $R_{\mu \nu}$. Using the relations previously obtained, we find that on physical states, $6 \mathrm{a}-6 \mathrm{~b}),(6 \mathrm{~d}-6 \mathrm{6t})$, and $(6 \mathrm{n})$ are verified, with the above mentioned values of $a_{i}$ 's. Thus (49) is indeed the correct identification. In fact, this relation should be expected. After twisting, the Lorentz algebra is isomorphic to $S U_{L^{\prime}}(2) \otimes S U_{R}(2)$ and thus some hybridization of the internal symmetry with the old Lorentz generators $J_{\alpha \beta}$ is expected.

\section{HERMITICITY AND EXCITED STATES}

As shown in the last section, TYM theory in flat Euclidian spacetime realizes the $S O(4)$ "Lorentz" algebra in such a way that the boost generators $M_{0 i}$ are non-hermitian (neither are they antihermitian). In order to classify the possible states of the theory, we wish to identify the unitary representations of the symmetry algebra. Let us concentrate here on the compact sector $S O(4)$. As is well known, the irreducible and unitary representations are in that case finite dimensional (dimension $\left(2 \ell_{1}+1\right)\left(2 \ell_{2}+1\right)$ with $\left.\ell_{1}, \ell_{2}=0,1,2 \ldots\right)$, the generators are represented by hermitian matrices and the group elements related to the identity can be written as $e^{i \alpha_{\mu \nu} M^{\mu \nu}}$ with parameters $\alpha_{\mu \nu}$. Now if $M_{0 i}$ is not hermitian, it is clear that as far as $S O(4)$ is concerned, the only admissible unitary representation will be the trivial one, in which $M_{0 i}=0$. The $S O(3)$ subgroup of spatial rotations generated by $M_{k j}$ does not suffer this problem, and the Hilbert space of the theory could carry the usual labels $\ell m$ of the $S O(3)$ representation since this subgroup commutes with $P_{0}$. But because $M_{0 i}$ are not hermitian, only $\ell=m=0$ will be present in that case. This can be seen in the

algebra: acting with both sides of (19) on the representation space will give the same result provided $M_{i j}$ is also vanishing.

If TYM is considered in Minkowski spacetime, with $g_{\mu \nu}=\eta_{\mu \nu}, M_{0 i}$ will also be non- 
hermitian, with equally dramatic consequences. Suppose we are interested in the unitary representations of the twisted algebra (6), assumed to be rotated to Minkowski metric, as specified in section [II]. To investigate them, we make use, as in the case of the superPoincaré algebra [7], of Wigner's method of induced representations [19]. This method is also appropriate here since our symmetry group possesses the same abelian invariant subgroup, namely the translations. In this method, one first makes a choice of "standard vector", eigenstates of $P_{\mu}$ and a representative member of the possible classes of eigenvalues of the Casimir $P_{\mu}^{2}$. One then identifies the little group, formed by the generators that leave the standard vector intact, and excluding the abelian subgroup. Once the irreducible unitary representations of the little group have been identified (restricting to finite dimensional ones), they are then used to induce an irreducible unitary representation of the whole group. This is done by acting on the standard vector with the generators that change its eigenvalue of $P_{\mu}$. These infinite dimensional representations then form the plane-wave basis, to which particles are associated.

Consider the massless case. The little supergroup is formed by $C_{1} \equiv M_{10}+$ $M_{13}, C_{2} \equiv M_{20}+M_{23}, M_{12}, Q, \bar{Q}_{\alpha}, S_{\mu \nu}, R_{\mu \nu}$. Acting with any of these will leave the vector $\left|p_{0}^{\mu}=(m, 0,0, m)\right\rangle$ unrotated. Now since

$$
\begin{gathered}
{\left[C_{1}, M_{12}\right]=-C_{2},} \\
{\left[C_{2}, M_{12}\right]=C_{1},} \\
{\left[C_{1}, C_{2}\right]=0,}
\end{gathered}
$$

is the Lie algebra $E_{2}$, and since we seek a finite dimensional representation, we are led to $C_{1}=C_{2}=0$ when acting on the standard vector, just as in the superPoincaré algebra [7]. Thus, at this level, the non-hermiticity of $M_{10}$ and $M_{20}$ appears irrelevant. However, in order to induce a representation of the entire group, we need a unitary realization of the finite transformation generated by $M_{30}, M_{10}-M_{13}$ and $M_{20}-M_{23}$. But with $M_{30}$ non-hermitian, this can only be implemented through a trivial realization: $M_{03}=0$. This in turn implies that if we consider the first part of Eq. (17) and choose $i=3$ when acting on $\left|p_{0}\right\rangle$, the LHS 
will vanish, and lead to $P_{3}\left|p_{0}\right\rangle=0$. (We refer to euclidean commutators for convenience; at this point the results clearly do not depend on the signs appearing in them.) One thus conclude that massless excitations will not occur in TYM.

A similar situation occurs if one attempts to construct massive representations. Taking as the standard vector $\left|p_{1}^{\mu}=(m, 0,0,0)\right\rangle$, the little group is made of $\left(M_{k j}, J_{k j}, Q, \bar{Q}_{\alpha}, S_{\mu \nu}, R_{\mu \nu}\right)$. Inducing a representation of the whole group will require a unitary operator for finite boosts, again this is only possible if the action of $M_{0 i}$ is trivial: $M_{0 i}\left|p_{1}\right\rangle=0$. But using now the first part of (18), we find $P_{0}\left|p_{1}\right\rangle=0$, again contradicting the assumption on $\left|p_{1}\right\rangle$. In this way, we recover, in a group theoretical context, the absence of dynamics in TYM.

We now focus on the last possibility : null representations with standard vector $\left|p_{3}^{\mu}=0\right\rangle$. (We will not consider spacelike representations). This vector is left unchanged by any Lorentz transformation and the little group is made of all the generators: $M_{\alpha \beta}, J_{\alpha \beta}, Q$, $\bar{Q}_{\alpha}, S_{\alpha \beta}$ and $R_{\alpha \beta}$. Here, representations of the full group and the little group coincide. As before, because we seek unitary representations, we will require that $M_{0 i}\left|p_{3}\right\rangle=0$. When used in (19) we obtain $M_{k j}\left|p_{3}\right\rangle=0$, showing the rotational invariance of $\left|p_{3}\right\rangle$, which has thus the characteristics of a vacuum state. Turning now to the action of $Q$, consider the time component of (33), it reads:

$$
\left\{Q, Q^{\dagger}\right\}\left|p_{3}\right\rangle=0
$$

since $\left|p_{3}\right\rangle$ is by construction a physical state. Projecting on $\left\langle p_{3}\right|$, we find

$$
\left\langle Q p_{3} \mid Q p_{3}\right\rangle+\left\langle Q^{\dagger} p_{3} \mid Q^{\dagger} p_{3}\right\rangle=0
$$

and conclude that $Q\left|p_{3}\right\rangle=Q^{\dagger}\left|p_{3}\right\rangle=0$.

Similarly, we can easily determine that the other generators have eigenvalue 0. By (34), $\bar{Q}\left|p_{3}\right\rangle=0$. Making use of (28), we then find $\bar{Q}_{i}\left|p_{3}\right\rangle=0$. Applying the same reasoning with (41) and (47), we find $S_{0 i}\left|p_{3}\right\rangle=0$ and $R_{0 i}\left|p_{3}\right\rangle=0$. Thus, all generators act trivially in TYM.

Now as mentioned before, the Lagrangian in (1) can be obtained by gauge fixing of a topological symmetry . The BRST charge introduced in that construction is the scalar $Q$ 
given in (21). In that context, the physical states are assumed to be annihilated by $Q$, and such that they are not of the form $Q|\alpha\rangle$. Having shown the former, we now argue for the latter, following Ref. [2]. Consider a state $|\psi\rangle=Q|\alpha\rangle$, with $P_{0}|\psi\rangle=0$. Because $\left[P_{0}, Q\right]=0$, $|\psi\rangle$ and $|\alpha\rangle$ can be chosen to have the same eigenvalue under $P_{0}$. But with $P_{0}|\alpha\rangle=0$, applying the steps given in (51) and (52) will lead to $|\psi\rangle=0$. We thus obtain, in the context of twisted $N=2$ SYM, the BRST cohomology condition of Refs. 24 [4 on physical states.

\section{CONCLUSION}

We have used the Hamiltonian formalism to study the symmetries of (11). This formalism offers the inconvenience of a non manifest covariance, but made explicit the generators, as well as the "propagation" of the Gauss law constraint through the algebra. In this context, it would be interesting to see how the algebra we have obtained is modified by the gauge fixing of the Yang-Mills symmetry [18]. We were also able to make precise the relation between the Lorentz generators of TYM $\left(M_{\alpha \beta}\right)$ and the twisted version of Lorentz and $S U_{I}(2)$ generators of $\operatorname{SYM}\left(J_{\alpha \beta}\right.$ and $R_{\alpha \beta}$ respectively) as displayed in (49). It is usually not illuminating to add symmetries to obtain new ones, but the interest here lies in their physical significance. One could avoid introducing the non-hermitian $M_{0 i}$. But in order to understand the Lorentz structure of the various objects (fields, charges, etc ) of the theory, they are needed. It is thus more sensible to discard $J_{\alpha \beta}$, keeping $M_{\alpha \beta}$ and $R_{\alpha \beta}$. In this way, $R_{\alpha \beta}$ appears as a symmetry of (目) unappreciated in previous work. In fact, its existence may seem odd at first sight, in view of the Coleman-Mandula theorem [20]. But as we have shown in Section $\mathbb{I \nabla}$, no massive unitary representations are realised in TYM, and in this way, the conclusions of the theorem are inapplicable. Nevertheless, more could be learned about $R_{\alpha \beta}$. Extending to more general manifolds, is it preserved [12]? Can it be used, along the lines of [16], to draw conclusions on the quantum theory at all orders in perturbation theory by restricting the possible counterterms (provided anomalies are absent)? It would 
also be interesting to investigate the extent of that symmetry in other topological theories. For instance, the symmetry algebra of the Chern-Simons theory in the Laudau gauge has

been found to coincide with a twisted $N=4$ superalgebra [17,21]. It is expected that a twisted internal symmetry will also exist in that case.

\section{ACKNOWLEDGMENTS}

I thank Roger Brooks for suggesting this investigation, and providing direction along the way. I also benefitted from comments from Daniel Freedman, Roman Jackiw, Kenneth Johnson, Choonkyu Lee and Claudio Lucchesi. 


\section{REFERENCES}

[1] For a review, see D. Birmingham, M. Blau, M. Rakowski and G. Thompson, Phys. Rep. 209 (1991) 129.

[2] E. Witten, Comm. Math. Phys. 117 (1988) 353.

[3] R.Brooks, D.Montano and J.Sonnenschein, Phys. Lett. B214 (1988) 91.

[4] L.Baulieu and I.M.Singer, Nucl. Phys. (Proc. Suppl.) 5B (1988) 91.

[5] J.P.Yamron, Phys. Lett. B213 (1988) 325.

[6] A.Karlhede and M.Roček, Phys. Lett. B212 (1988) 51.

[7] P.West, Introduction to Supersymmetry and Supergravity (2nd ed. World Scientific, 1990).

[8] S.J.Gates, M.T.Grisaru, M.Roček and W.Siegel, Superspace (Frontiers in Physics, Benjamin Cummings, 1983).

[9] M.F.Sohnius, Phys. Rep. 128 (1985) 39.

[10] R.Brooks, Phys. Lett. B267 (1991) 481

[11] R.Brooks and D.Kastor, Phys. Lett. B246 (1990) 99.

[12] A.Galperin and O.Ogievetsky, Phys. Lett. B236 (1990) 33.

[13] S.B. Treiman, R. Jackiw, B. Zumino and E.Witten, Current Algebra and Anomalies (Princeton Series in Physics, 1985), see R. Jackiw in Field Theoretic Investigations in Current Algebra.

[14] C.Nash and S.Sen, Topology and Geometry for Physicists (Academic Press, 1983).

[15] H.D Dahmen, S. Marculescu and L. Szymanowski, Nucl. Phys. B383, (1992) 110; Phys. Lett. B252 (1990) 591. 
[16] M. Werneck de Oliveira, Phys. Lett. B307 (1993) 347.

[17] F.Delduc, F.Gieres and S.P.Sorella, Phys. Lett. B225 (1989) 367.

[18] S.Ouvry, R.Stora and P.Van Baal, Phys. Lett. B220 (1989) 159.

[19] E.P. Wigner, Ann. of Math. 40 (1939) 149.

[20] S. Coleman and J. Mandula, Phys. Rev. 159 (1975) 1251.

[21] R. Brooks, J.-G. Demers and C. Lucchesi, MIT-CTP preprint 2237, hep-th 9308127. 